\title{
The Design and Improvement of a Fuzzy Controller Based on a Single Inverted Pendulum
}

\author{
Xiuyun $\mathrm{Li}^{1, \mathrm{a}}$, Yanjun Ren ${ }^{2}$ \\ 1.Chongqing Vocational Institute of Engineering, Chongqing 402260, China \\ 2.Chongqing Technology and Business Institute, Chongqing 400052, China
}

a.973736111@qq.com

Keywords: Inverted pendulum system; Nonlinear mathematical model; Fuzzy Controller

\begin{abstract}
This paper has introduced the main characteristic and some current re-searches about the inverted pendulum system. A nonlinear mathematical model of invert-ed pendulum is established based on the newtonian dynamics method. Then the design of traditional fuzzy controller is presented, the result of simulation shows the dynamic performance is not so good. Finally, the improvement of the traditional fuzzy controller is presented, the result of simulation shows the improved fuzzy controller gets a better dynamic performance than the traditional fuzzy controller.
\end{abstract}

\section{Introduction}

The Significance of research papers. Inverted pendulum behaves some characteristics such as instability, nonlinearity and strong coupling. Inverted pendulum control study can effectively respond to a great many classical problems, like nonlinear control problems, robustness issues, and mobility and tracking issues, etc. Therefore, the inverted pendulum control is a classic instance of the control field to research control algorithm [1,2]. Inverted pendulum control method can be applied to the military, aerospace and many other aspects, for instance, a spacecraft balance control, the robot posture control and two-wheeled self-balancing vehicle balance control, etc. Weaver et al. [3] even have determined the degree of applicability of the inverted pendulum model is suitable for stooping and crouching postures control in 2014.

Research Actuality. Characteristic of the inverted pendulum invoked great interest in scholars all over the word. It starts the research of single inverted pendulum system as early as the 1960s. In the late 1960s, the concept of an inverted pendulum is proposed. In 1972, Sturgeon has successfully achieved the stability control of double inverted pendulum, Furuta [4] has been successfully completed the stable control of double inverted pendulum via linear control simulation method in 1978. In China, researchers start to research the inverted pendulum since the 1980s. Xi'an Jiaotong University successfully completed the stable control of double inverted pendulum by optimal control and reduced order observer of modern control theories in 1982; Shanghai Mechanical Engineering College has successfully completed research of single and double inverted pendulum system, and achieved the control of double inverted pendulum on an inclined orbit based on this in 1987.

Researchers interested in intelligent control method as more and more effective algorithms are submitted. Neural networks, fuzzy control, genetic algorithm, anthropomorphic intelligent control and expert systems have penetrated into the control field of various types of inverted pendulum. Andorson [5] successfully controlled a pendulum in 1988 via self-learning fuzzy neural network control instead of traditional control methods. Zhou [6] has solved the stability control problem of the simple pendulum by selecting the neural network control rule as the foundation of control algorithm. Xu et al. [7] submitted fuzzy control method based on variable structure, and based on this they have successfully finished the stability control. Li [8] achieved stability control of triple inverted pendulum in September, 2001, and finished the stability control of 
Level 4 inverted pendulum. Sun [9] has achieved the control of double inverted pendulum by the RNA genetic algorithm of the type-2 fuzzy logic systems optimization.

For traditional fuzzy control, the rules of control obtained by experiments is not optimal usually, the dynamic performance may behave as large overshoot , long accommodation time, etc.

This paper applies an alterable quantification factor and scale factor method according to the inputs of the fuzzy controller based on the process of design of traditional fuzzy controller. The result of the simulation shows the dynamic performance of the single inverted pendulum can be improved.

\section{Modeling and performance analysis of single inverted pendulum}

Model of the inverted pendulum. Fig. 1 and Fig. 2 show the force analysis of inverted pendulum system and rod of pendulum.

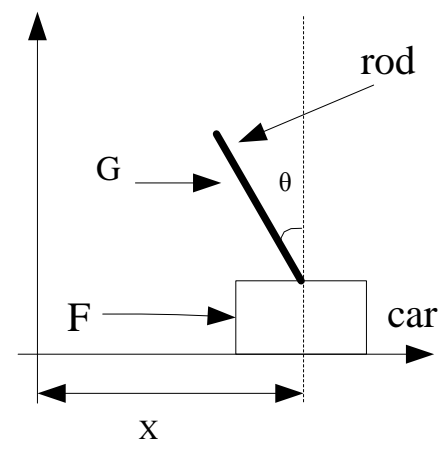

Fig. 1 force analysis of inverted pendulum system

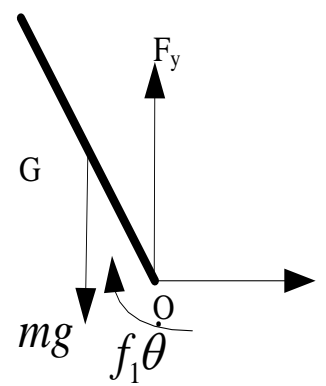

Fig. 2 force analysis of rod

For concision of writing, all the symbols represent the parameters are described below:

Nomenclature

m0 quality of the car

1 distance of $\mathrm{O}$ to $\mathrm{G}$

$\mathrm{ml}$ quality of the rod

$\mathrm{L}$ length of rod

$\mathrm{O}$ the coordinate of Rotation Point

$\mathrm{J}$ moment of inertia

$\mathrm{G}$ the coordinate of the centroid of rod

F external force

f0 COF between car and guide rail

$\theta$ The angle between the pendulum and the vertical upward direction

From Fig. 1 and Fig. 2, the horizontal force of rod can be written as

$F_{x}=m_{1}(x-l \sin \theta) "$

Namely

$$
F_{x}=m_{1} \ddot{x}-m_{1} l \ddot{\theta} \cos \theta+m_{1} l \dot{\theta}^{2} \sin \theta
$$

Torque balance equation of rod is

Namely

$$
\left(J+m_{1} l\right) \ddot{\theta}=m_{1} g l \sin \theta-f_{1} \dot{\theta}+m_{1}\left(O_{x}\right) " l \cos \theta-m_{1}\left(O_{y}\right) " l \sin \theta
$$

The horizontal force of rod is

$$
\left(J+m_{1} l\right) \ddot{\theta}=m_{1} g l \sin \theta-f_{1} \dot{\theta}+m_{1} \ddot{x} l \cos \theta
$$

$$
F-f_{0} \dot{x}-F_{x}=m_{0} \ddot{x}
$$

From (2) and (5), $F_{x}$ could be Canceled

$$
\left(m_{0}+m_{1}\right) \ddot{x}-m_{1} l \ddot{\theta} \cos \theta=-f_{0} \dot{x}-m_{1} l \dot{\theta} \sin \theta+F
$$

According to (4) and (6), differential equations of single inverted pendulum can be described as

$$
\left\{\begin{array}{l}
a_{0} \ddot{x}-a_{1} \ddot{\theta} \cos \theta=-a_{1} \dot{\theta}^{2} \sin \theta-f_{0} \dot{x}+u \\
-a_{1} \ddot{x} \cos \theta+b_{1} \ddot{\theta}=-f_{1} \dot{\theta}+a_{1} g \sin \theta
\end{array}\right.
$$

where $a_{0}=m_{0}+m_{1}, a_{1}=m_{1} l, b_{1}=J+m_{1} l^{2}, u=F$ 
Rewrite (7) as the matrix form

$$
\left[\begin{array}{cc}
a_{0} & -a_{1} \cos \theta \\
-a_{1} \cos \theta & b_{1}
\end{array}\right]\left[\begin{array}{l}
\ddot{x} \\
\ddot{\theta}
\end{array}\right]=\left[\begin{array}{cc}
-f_{0} & -a_{1} \dot{\theta} \sin \theta \\
0 & -f_{1}
\end{array}\right]\left[\begin{array}{l}
\dot{x} \\
\dot{\theta}
\end{array}\right]+\left[\begin{array}{l}
0 \\
a_{1} g \sin \theta
\end{array}\right]+\left[\begin{array}{l}
1 \\
0
\end{array}\right] u
$$

This is the nonlinear mathematical expression of inverted pendulum system.

Analysis of the model. Let $m_{0}=0.618 \mathrm{~kg}, m_{1}=0.0737 \mathrm{~kg}, L=0.350 \mathrm{~m}, l=0.1225 \mathrm{~m}$, linearization of (8) can be written as

$$
\left[\begin{array}{c}
\dot{x} \\
\ddot{x} \\
\dot{\theta} \\
\ddot{\theta}
\end{array}\right]=\left[\begin{array}{cccc}
0 & 1 & 0 & 0 \\
0 & 0 & 0 & 0 \\
0 & 0 & 0 & 1 \\
0 & 0 & 60 & 0
\end{array}\right]\left[\begin{array}{c}
x \\
\dot{x} \\
\theta \\
\dot{\theta}
\end{array}\right]+\left[\begin{array}{c}
0 \\
0 \\
0 \\
6.122
\end{array}\right] u, y=\left[\begin{array}{c}
x \\
\theta
\end{array}\right]=\left[\begin{array}{llll}
1 & 0 & 0 & 0 \\
0 & 0 & 1 & 0
\end{array}\right]\left[\begin{array}{c}
x \\
\dot{x} \\
\theta \\
\dot{\theta}
\end{array}\right]
$$

Thus we can know that $\operatorname{rank}\left(\left[\begin{array}{llll}B & A B & A^{2} B & A^{3} B\end{array}\right]\right)=4$ and $\operatorname{rank}\left(\left[\begin{array}{llll}C & C A & C A^{2} & C A^{3}\end{array}\right]\right)=4$ easily. so the system is both controllable and observable.

\section{Trditional fuzzy controller design}

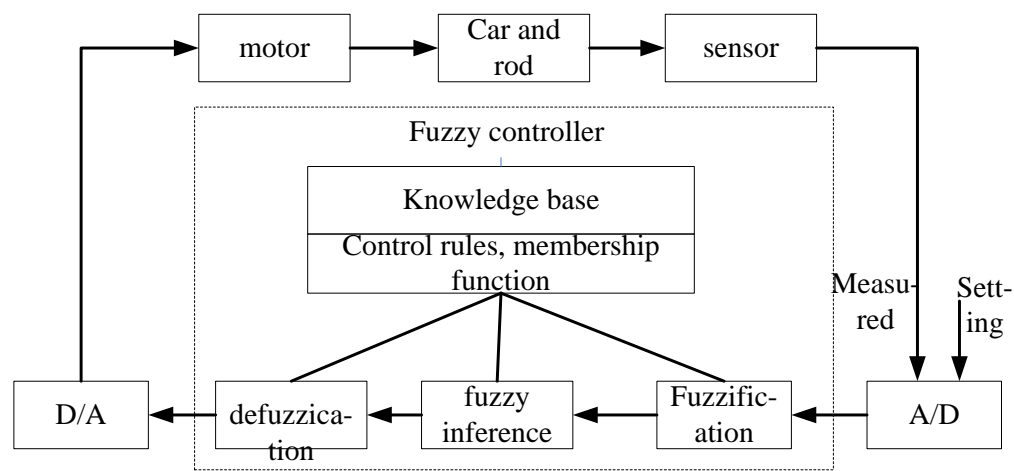

Fig. 3 block diagram of Fuzzy control

Based on the model (8), this part will design a fuzzy controller to steady the inverted pendulum system.

Fig. 3 is the block diagram when fuzzy controller is added in the inverted pendulum system.

In Fig. 3, when the setting value is balance point, both the displacement of the car and the angle of the rod are zeros. Sensors measure the displacement of car and the angle of the rod, which is feedback to the set value, forming bias, as the fuzzy controller input. Fuzzy controller translates the input variables into fuzzy variables based on fuzzy subset and membership functions, and exports fuzzy outputs via fuzzy reasoning process. After the defuzzification, explicit control variable can be obtained, to reduce the error between setting value and real value.

A single inverted pendulum has four inputs, the displacement of the car, the angle of the rod, the car speed, and the angular velocity of the pendulum. If the amount of each input domain is divided into seven fuzzy sets, then there exists $7^{4}=2401$ control rules, this can cause difficulty to the design of the controller. Refer to $[10,11]$, this problem can be solved by the relevant fusion function.

According to the relevant characteristic of single inverted pendulum, $\theta$ and $\dot{\theta}$ are selected as the principal elements, relevant merger coefficients are $k_{\theta}$ and $k_{\dot{\theta}}$. Let output variable $\tilde{X}=[E, E C]^{T}$

$$
\begin{aligned}
u & =k_{x} \cdot x+k_{\theta} \cdot \theta+k_{\dot{x}} \cdot \dot{x}+k_{\dot{\theta}} \cdot \dot{\theta} \\
& =k_{\theta}\left(\frac{k_{x}}{k_{\theta}} \cdot x+\frac{k_{\theta}}{k_{\theta}} \cdot \theta\right)+k_{\dot{\theta}}\left(\frac{k_{\dot{x}}}{k_{\dot{\theta}}} \cdot \dot{x}+\frac{k_{\dot{\theta}}}{k_{\dot{\theta}}} \cdot \dot{\theta}\right)
\end{aligned}
$$


Therefore

$$
E=\frac{k_{x}}{k_{\theta}} \cdot x+\frac{k_{\theta}}{k_{\theta}} \cdot \theta, E C=\frac{k_{\dot{x}}}{k_{\dot{\theta}}} \cdot \dot{x}+\frac{k_{\dot{\theta}}}{k_{\dot{\theta}}} \cdot \dot{\theta}
$$

The output of the fusion function can be obtained as

$$
\tilde{X}=\left[\begin{array}{cccc}
\frac{k_{x}}{k_{\theta}} & 0 & \frac{k_{\theta}}{k_{\theta}} & 0 \\
0 & \frac{k_{\dot{x}}}{k_{\dot{\theta}}} & 0 & \frac{k_{\dot{\theta}}}{k_{\dot{\theta}}}
\end{array}\right]\left[\begin{array}{c}
x \\
\dot{x} \\
\theta \\
\dot{\theta}
\end{array}\right]
$$

The specific values of $k_{\theta}$ and $k_{\dot{\theta}}$ can be obtained by Linear Quadratic Optimal Control method.

Thus, four-dimensional input vector can be simplified by two-dimensional vector.

The domain of discourse of the input variables E, EC and $\mathrm{u}$ are all selected as $[-6,6]$, in this domain seven fuzzy sets are selected: $N B 、 N M 、 N S 、 Z E \quad P S \quad P M \quad R B \quad$. To reduce the compute load, membersh- ips of $E, E C, u$ are all selected as triangle membership function.

Refer to continuous experiments, rules of fuzzy control is shown as below.

\begin{tabular}{|c|c|c|c|c|c|c|c|}
\hline $\mathrm{E}^{\mathrm{EC}}$ & $\mathrm{NB}$ & $\mathrm{NM}$ & NS & $\overline{Z E}$ & PS & $\mathrm{PM}$ & PB \\
\hline $\mathrm{NB}$ & $\mathrm{NB}$ & NB & $\mathrm{NB}$ & NM & NM & NS & ZE \\
\hline $\mathrm{NM}$ & NB & NB & $\mathrm{NM}$ & $\mathrm{NM}$ & NS & ZE & PS \\
\hline NS & NB & NM & $\mathrm{NM}$ & NS & $\mathrm{ZE}$ & PS & $\mathrm{PM}$ \\
\hline$\overline{\mathrm{ZE}}$ & NM & NM & NS & $\mathrm{ZE}$ & PS & $\mathrm{PM}$ & $\mathrm{PM}$ \\
\hline$\overline{\mathrm{PS}}$ & NM & NS & $\mathrm{ZE}$ & PS & PM & $\mathrm{PM}$ & PB \\
\hline $\mathrm{PM}$ & NS & ZE & PS & PM & PM & PB & PB \\
\hline PB & $\mathrm{ZE}$ & PS & PM & PM & PB & PB & PB \\
\hline
\end{tabular}

Table 1 fuzzy control rules of inverted pendulum

The design of the controller is fulfilled nearly, the rest work is the choice of the quantization factor and scale factor. This can be adjusted in experiments. Result of Simulation is shown in Fig. 4.

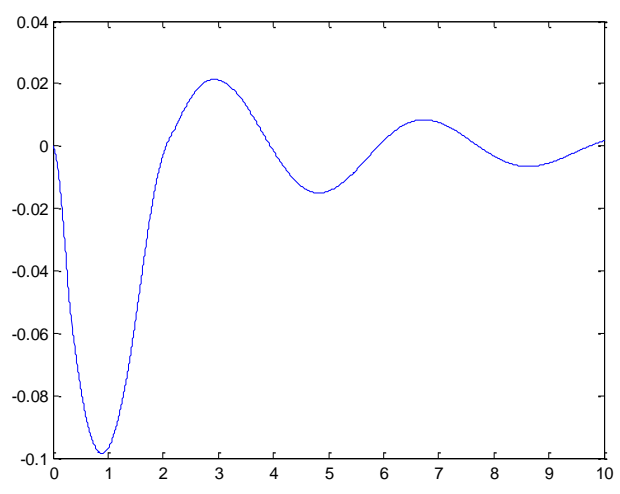

(a) Displacement of car

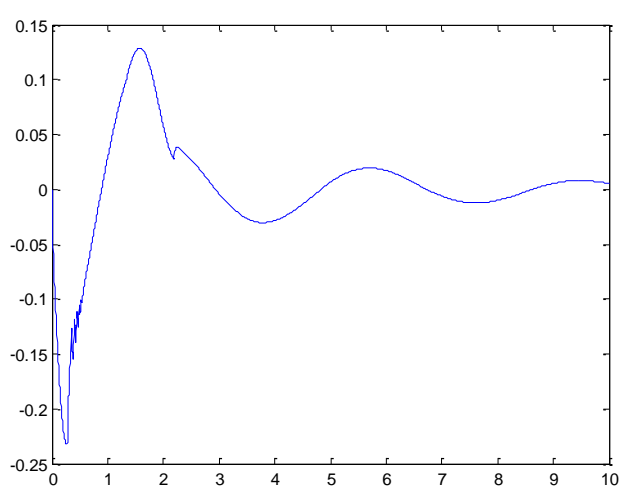

(b) Speed of car 


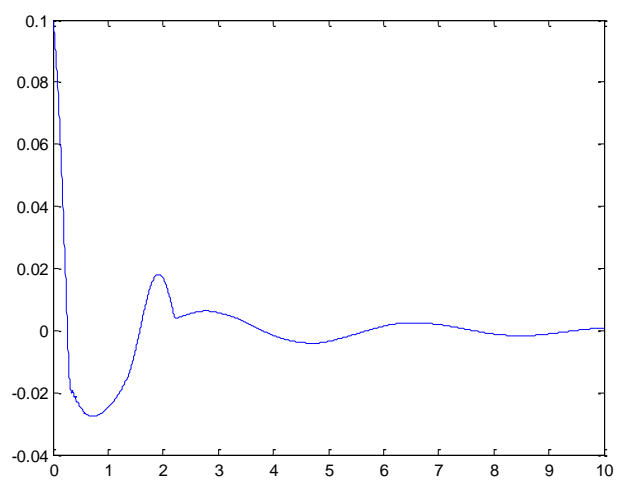

(c) Angle of rod

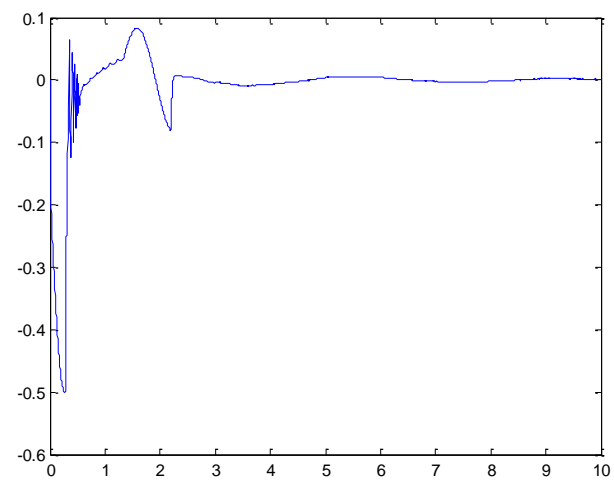

(d) The angular velocity of the rod

Fig. 4 Result of the single inverted pendulum system

\section{Improved fuzzy controller design}

As is shown in Fig. 4, the dynamic performance of the controller we designed is not well enough. This part will provide a scheme to ameliorate the dynamic performance of the controller. For quantization factor and scale factor play an import role on the performance of the fuzzy controller.

Refer to the analysis of [12]. We know that quantification factor $k_{u}$ should be drastically reduced when system is instable. When the system is marginally stable, $k_{u}$ could be appropriately reduced. When steadystate error exists, $k_{u}$ should be increased appropriately. And increase scale factor $k_{e}$ slightly at the same time. When transition time perform too long, reduce $k_{e c}$ appropriately, and $k_{e c}$ should be increased slightly when the overshoot is too large. According to this, a fuzzy controller can have multiple sets of quantization factor and scale factor, these factors change with the dynamic performance of the system. Therefore, we can set a threshold, when the input exceeds the threshold, the quantization factor and scale factor will change into another group. Here we choose the threshold value as the position of car, when the absolute value of position is less than $s_{0}=3$, quantization factor and scale factor are selected as $k_{e}=8, k_{e} c=4, k_{u}=35$, and when the absolute value of Position is larger than $s_{0}$, these parameters are selected as $k_{e}=10, k_{e} c=5, k_{u}=23$, the results of the improved fuzzycontroller based on the alterable parameters are shown in Fig. 6.

Compared to Fig. 5, it is obvious that the overshoot decay faster and the settling time become shorter. The dynamic performance is more excellent, the improvement of the controller is effective.

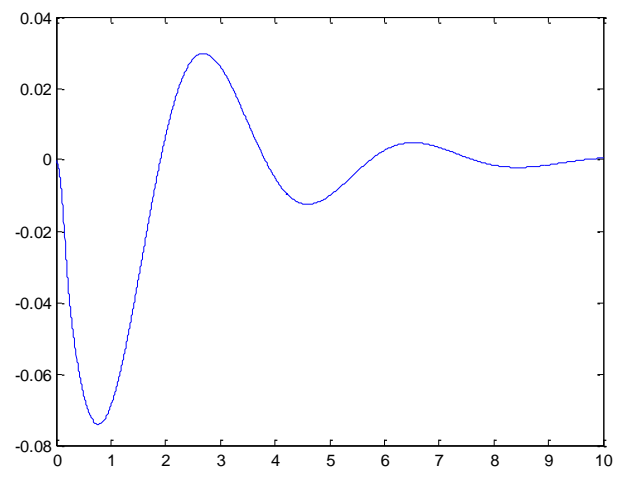

(a) Displacement of car

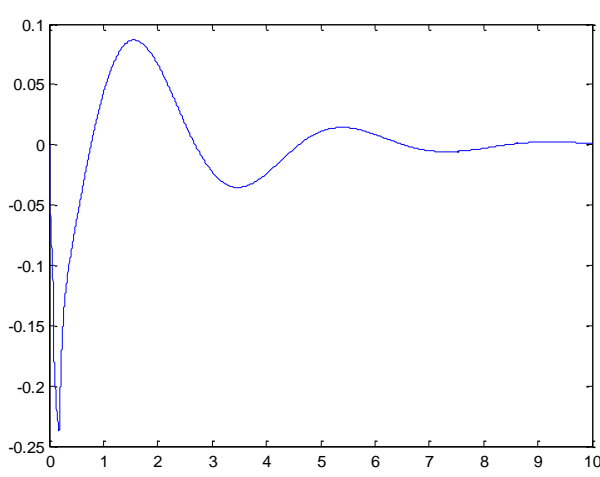

(b) Car velocity 


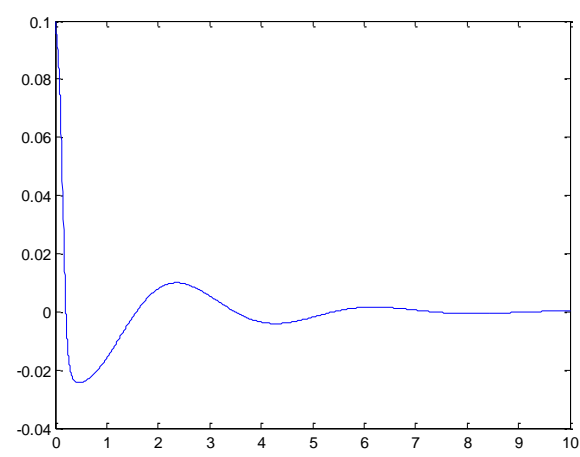

(c) Angle of rod

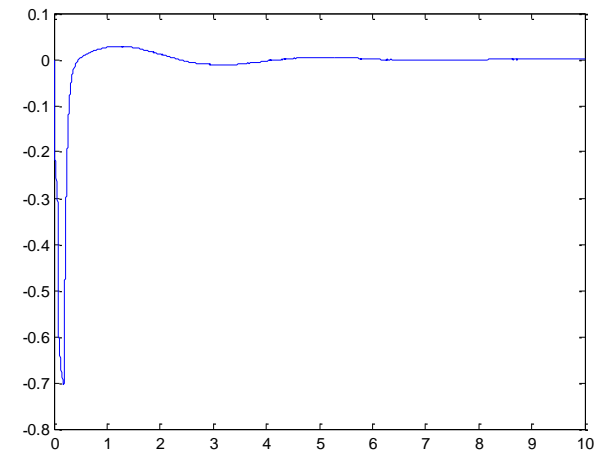

(d) The angular velocity of the rod

Fig. 5 The results of alterable quantization factor and scale factor

\section{Conclusions}

The fuzzy control method can steady the instable a single inverted pendulum system, significant factors are the fuzzy control rules, scale factor and quantization factor. The control rules is the kernel of a fuzzy controller, we optimize the control rule by repeatable experiments. The scale factor and quantization factor influence the dynamic performance of the inverted system. If these two factors could vary from the state of the system, the dynamic performance of the system would be optimized without doubt, the results of the simulation about Self-adjusting of scale factor and quantization factor method in part 4 have verified this.

\section{Acknowledgement}

This work was financially supported by the Natural Science Foundation of Chongqing Municipal Educat ion Commission, China (Grant No. KJ1403209).

\section{References}

[1] H. Heng. Inverted pendulum control and parameter optimization based on MATLAB [D]. Wuhan University of Science and Technology, 2008.

[2] J. Yi, N. Yubazaki, K. Hirota. A new fuzzy controller for stabilization of parallel-type double inverted pendulum system [J]. Fuzzy Sets and Systems, 2002, 126(1): 105-119.

[3] T. B. Weaver, M. N. Glinka, A. C. Laing. Moving beyond quiet stance: Applicability of the inverted pendulum model to stooping and crouching postures [J]. Journal of Biomechanics, 2014, 47(14): 35743579 .

[4] K. Furuta, T. ochiai and N. Ono. Attitude control of a triple inverted pendulum. International Journal of Control, 1984, 39(6): 1351-1365.

[5] W. Andorson. Learing to Control an Inverted Pendulum Using neural Networks. IEEE Control System Magazine, 1989, 9(3): 31-36.

[6] J. Zhou. Control rules of neural network to solve the problem of inverted pendulum balance based on BP [C]. Proceedings of Chinese Control and Decision Conference, 2011.

[7] H. Xu, Z. Sun, F. Sun. Studies of fuzzy neural sliding mode control based on double inverted pendulum

[C]. Proceedings of China Intelligent Automation Conference, 2014. 
[8] H. Li. Variable universe adaptive fuzzy controller of four level inverted pendulum [J]. Chinese Science, 2002, 32(1): 65-75.

[9] Z. Sun, N. Wang, Y. Bi. Type-1/type-2 fuzzy logic systems optimization with RNA genetic algorithm for double inverted pendulum [J]. Applied Mathematical Modelling, 2015, 39(1): 70-85.

[10] Z. Chen. Fuzzy Control of Double Inverted Pendulum System [D]. Northeastern University, 2008.

[11] S. Wei. Fuzzy control of single inverted pendulum [D]. Taiyuan University of Science and Technology, 2012.

[12] X. Shi. Fuzzy control and simulation based on MATLAB [M]. Tsinghua University Press, 2008. 